\title{
Caractérisation de la végétation des clairières sur sol hydromorphe du Parc National de Lobéké, Est-Cameroun
}

\author{
Lucie Félicité TEMGOUA $^{1 *}$, Marie Caroline MOMO SOLEFACK ${ }^{2}$, \\ Max-Vittel MEVOUNGOU ${ }^{1}$ et Achile MENGAMENYA ${ }^{3}$ \\ ${ }^{1}$ Université de Dschang, Département de Foresterie, Faculté d'Agronomie et de Sciences Agricoles, \\ B.P. 222, Dschang, Cameroun. \\ ${ }^{2}$ Université de Dschang, Département de Biologie Végétale, Faculté de Science B.P. 67, Dschang, \\ Cameroun. \\ ${ }^{3}$ Ministère des Forêts et de la Faune, Parc National de Lobéké, Cameroun. \\ *Auteur correspondant ; E-mail: temgoualucie@yahoo.fr
}

\section{RESUME}

Le Parc National de Lobéké est caractérisé par la présence des clairières sur sol hydromorphe dont la végétation est très importante pour la grande et la moyenne faune herbivore. Dans le but de contribuer à l'aménagement de ces clairières, une caractérisation de leur végétation a été faite. L'inventaire floristique a été réalisé dans quatre clairières en utilisant la méthode phytosociologique sigmatiste de Braun-Blanquet. Le dispositif expérimental était constitué, au total, de 850 quadrats de $1 \mathrm{~m}^{2}$ chacun. Dans chacun des quadrats, tous les individus ont été comptés. Les résultats montrent que 70 espèces végétales appartenant à 60 genres répartis dans 29 familles ont été identifiées dans l'ensemble des clairières. Le nombre d'espèces par clairière varie de 30 à 45. Les indices de diversité varient de 1,61 à 3,49 (Shannon), 0,55 à 0,93 (Simpson) tandis que l'équitabilité de Piélou varie de 0,33 à 0,73 . La diversité était plus grande à Djangui et plus faible à Djaloumbé. Les familles dominantes étaient les Cyperaceae, les Poaceae et les Fabaceae. Les espèces les plus fréquentes dans l'ensemble des clairières étaient Rhynchospora corymbosa, Cyperus difformis, Acroceras amplectens, Paspalum conjugatum, Pentodon pentandrus, Acmela caulirhiza, Commelina sp., Desmodium adscendens et Hydrocharis chevalieri. Les thérophytes et les géophytes étaient les formes de vies dominantes. La dominance des phanérophytes à Djaloumbé traduit la fermeture de cette dernière. Des actions d'enlèvement de Mimosa pudica et des Cyperaceae envahissantes devraient être envisagées dans le cadre de l'aménagement de ces clairières.

(C) 2018 International Formulae Group. All rights reserved.

Mots clés: clairières marécageuses, forêt dense, flore, parc national, Lobéké, Cameroun

\section{Characterization of clearings vegetation on hydromorphic soils in Lobéké National Park, Eastern Cameroon}

\begin{abstract}
The Lobéké National Park is characterized by the presence of clearings on hydromorphic soil, with a very important vegetation for large and medium herbivore fauna. In the view of contributing to the management of these clearings, a characterization of their vegetation has been made. The floristic inventory
\end{abstract}


was carried out in four clearings using the phytosociological sigmatist method of Braun-Blanquet. The experimental design consisted of 850 quadrats of $1 \mathrm{~m}^{2}$ each. In each of the quadrats, all the individuals were counted. The results show that 70 plant species belonging to 60 genera and 29 families were identified in all the clearings. The number of species per clearing varies from 30 to 45 . The indices of diversity of the clearings vary from 1.61 to 3.49 (Shannon), 0.55 to 0.93 (Simpson) while Piélou's evenness varies from 0.33 to 0.73 . The diversity was greater at Djangui and lower at Djaloumbe. The dominant families were Cyperaceae, Poaceae and Fabaceae. The most frequent species were Rhynchospora corymbosa, Cyperus difformis, Acroceras amplectens, Paspalum conjugatum, Pentodon pentandrus, Acmela caulirhiza, Commelina sp., Desmodium adscendens and Hydrocharis chevalieri. Therophytes and geophytes were the most abundant forms of life. The dominance of phanerophytes at Djaloumbé reflects the closure of this clearing. Actions to remove Mimosa pudica and invasive Cyperaceae should be undertaken in the context of the management of these clearings.

(C) 2018 International Formulae Group. All rights reserved.

Keywords: Swampy clearing, rain forest, flora, national park, Lobéké, Cameroon.

\section{INTRODUCTION}

Les massifs forestiers du Bassin du Congo sont constitués par endroits des formations herbeuses appelées clairières. Une clairière est un endroit dégarni d'arbres dans une zone boisée, où la lumière du soleil atteint le niveau du sol. Elle peut être installée sur la terre ferme (sols secs) ou sur des sols gorgés d'eau (Boupoya, 2010). Les clairières sur sol hydromorphe ou "Baïs" en référence à leur nom en langue Baka sont celles qui sont installées sur des sols gorgés d'eau. White et Edwards (2001) définissent une clairière sur sol hydromorphe comme une clairière dans la forêt, généralement localisée sur un cours d'eau, où de nombreux mammifères se concentrent pour absorber des sels minéraux ou consommer la végétation aquatique.

Les clairières sont des lieux de concentration des grands mammifères. Les espèces qui y sont présentes sont les gorilles de plaine (Gorilla gorilla gorilla), les éléphants de forêt (Loxodonta africana cyclotis), les buffles (Syncerus cafer nanus), les sitatungas (Tragelapus spekei), les hylochères (Hylocherus meinertzhageni), les potamochères (Potamochoerus porcus), ainsi que les oiseaux (Magliocca et Gautier-Hion, 2001; Eno-Nkuh, 2011). Les clairières fournissent aux espèces animales des ressources importantes telles qu'un sol, une eau et des espèces de plantes riches en minéraux (Magliocca et Gautier-Hion, 2001,
2002 ; Metsio Sienne et al., 2013). Ces clairières se présentent sous forme d'un tapis herbacé principalement composé d'espèces graminéennes. Elles peuvent être parsemées d'arbustes aux formes rabougries et de hautes touffes d'herbes (Boupoya, 2010). Selon Magliocca et Gautier-Hion (2001), le maintien de ces milieux serait justifié par l'hydromorphie des sols et l'effet du piétinement de certains animaux sauvages, notamment les ongulés, qui y empêchent la germination et le développement des arbustes et arbres. Les clairières en raison de leur ouverture, permettent une observation relativement aisée de la grande faune, offrant ainsi de bonnes opportunités de développement touristique. Sur le plan de la recherche, elles permettent aussi de conduire des études éthologiques difficiles à mener dans les zones forestières plus fermées.

Plusieurs études ont été menées sur la dynamique de fréquentation des clairières forestières en Afrique centrale. Il s'agit des études de Turkalo et al. (2013), Magliocca (2000) et Gatti et al. (2004) en République Centrafrique; Eno-Nkuh (2011), Noupa et Nkongmeneck (2008a) au Cameroun; Vande Weghe (2006), Mindonga et al. (2016) et Zinga et al. (2016) au Gabon et Gessner et al. (2013) dans les clairières du Trinational de la Sangha.

En ce qui concerne la végétation des clairières hydromorphes, quelques études ont 
été menées par Temgoua (2006) et Boupoya et al. (2010) au Gabon. Au Cameroun les études ont été menées par Noupa et Nkongmeneck (2008b) sur la diversité floristique des clairières sèches sur schiste du massif forestier de Boumba-Bek et Nki.

Le Parc National de Lobéké est caractérisé par la présence des clairières sur sols hydromorphes. $\mathrm{Si}$ des travaux d'inventaire botanique ont été globalement menés par Harris (1999) dans la zone du parc, aucune étude spécifique ne s'est intéressée aux clairières qui y sont présentes. L'objectif de cette étude était de caractériser la végétation de ces habitats particuliers du Parc National de Lobéké.

\section{MATERIEL ET METHODES \\ Présentation du site d'étude}

Le Parc National de Lobéké (PNL), créé en 2001, couvre une superficie d'environ 217854 ha. Il est situé entre les latitudes $2^{\circ} 05^{\prime}-2^{\circ} 30^{\prime} \mathrm{N}$ et les longitudes $15^{\circ} 33^{\prime}$ $16^{\circ} 11^{\prime} \mathrm{E}$ au Sud-Est du Cameroun (Figure 1). Le PNL fait partie du paysage du Tri-national de la Sangha (TNS) avec les parcs nationaux de Dzanga-sangha en République Centrafricaine et Nouabalé- Ndoki en République du Congo (Tsakem et al., 2015).

Le climat du PNL est typiquement équatorial avec quatre saisons. La grande saison de pluies s'étend de septembre à novembre alors que la petite saison de pluies va de mars à juin. La grande saison sèche dure de décembre à février et la petite saison sèche, de juillet à août. Les précipitations s'étalent tout au long de l'année avec deux pics en avril-mai et en septembre-octobre (Ekobo, 1995). La hauteur moyenne des précipitations se situe autour de $1500 \mathrm{~mm} / \mathrm{an}$. Les moyennes mensuelles des températures varient de $23{ }^{\circ} \mathrm{C}$ à $26{ }^{\circ} \mathrm{C}$ et l'humidité relative varie de 60 à 90\% (TNS, 2012). Le PNL, par sa localisation sur le versant Nord-Ouest de la cuvette congolaise, fait partie du Bassin du Congo avec des formations métamorphiques typiques d'âge Précambrien à Cambrien, appartenant à la série du Dja inferieur et composées essentiellement de tillites, quartzites, schistes, calcaires et dolérites (Nzooh, 2003). Son relief est relativement plat avec cependant quelques collines. L'altitude varie entre $400 \mathrm{~m}$ et 700 $\mathrm{m}$. Les sols sont indurés et recouverts par endroits de cuirasses ferrugineuses. Ce sont des sols ferralitiques dérivés des roches métamorphiques anciennes (Laclavère, 1979). Les sources de la rivière Lobéké à l'intérieur $\mathrm{du}$ parc entretiennent un complexe de clairières marécageuses. Sur le plan phytogéographique, la végétation du PNL est considérée comme transitoire entre la forêt sempervirente du Dja et la forêt semi-décidue à Sterculiaceae et à Ulmaceae (Letouzey, 1985). Les résultats des inventaires floristiques réalisés dans la zone de Lobéké attestent la présence de 764 espèces végétales appartenant à 102 familles (Harris, 1999). La répartition en type morphologique ressort une diversité élevée des espèces arborescentes et arbustives (440 espèces) par rapport à celle des autres groupes (161 espèces de lianes et 163 espèces d'herbacées).

\section{Echantillonnage}

Le choix des clairières retenues pour la présente étude a été guidé par deux critères à savoir d'une part les clairières qui font l'objet d'un suivi mensuel régulier et disposent d'un mirador pour les observations directes et indirectes éco-éthologiques et d'autre part, les clairières qui ont potentiellement des atouts pour le développement du tourisme de vision. Ainsi, quatre clairières ont été retenues sur les sept principales du parc. Il s'agit de Ndangayé, Djangui, Djaloumbé et Petite Savane. Le Tableau 1 présente les paramètres dimensionnels des différentes clairières.

L'étude de la diversité floristique a été conduite par sondage en utilisant la méthode d'échantillonnage systématique. A l'aide du logiciel DISTANCE, des transects linéaires équidistants de 40 mètres ont été matérialisés sur les cartes des clairières (Figure 2). Les parcelles de comptage de $3 \mathrm{~m} \times 3 \mathrm{~m}$ ont ensuite été matérialisées sur les transects. 


\section{Détermination de la diversité floristique et du recouvrement}

Une fois sur le terrain, le point de début de chaque transect a été considéré comme le centre de la première parcelle du transect. De ce centre, une distance de 50 mètres a été mesurée au moyen du double décamètre afin de trouver le centre de la parcelle suivante. Chaque parcelle de $3 \mathrm{~m} \times 3 \mathrm{~m}$ de surface a été divisée en 9 quadrats de $1 \mathrm{~m}^{2}$. L'inventaire de la végétation a été conduit sur cinq (5) quadrats choisis systématiquement dans l'ordre suivant: $1,3,5,7$ et 9 soit $5 \mathrm{~m}^{2}$ afin d'inventorier au moins $50 \%$ de la parcelle. Toutes les espèces végétales contenues dans une placette de $1 \mathrm{~m}^{2}$ ont été comptées pied par pied.

L'échelle mixte d'abondancedominance de Braun-Blanquet (1932) a été utilisée pour l'estimation de l'importance relative des espèces. A chacune des espèces identifiées dans un relevé, a été attribué un coefficient d'abondance-dominance. Ces valeurs semi-quantitatives ont ensuite été transformées en unités quantitatives en utilisant les correspondances de Dufrêne (2003). L'échelle pour la quantification du recouvrement moyen (RM) se dessine ainsi qu'il suit :

5 : espèces recouvrant 75 à $100 \%$ de la superficie du relevé $(\mathrm{RM}=87,5 \%)$;

4 : espèces recouvrant 50 à $75 \%$ de la superficie du relevé $(\mathrm{RM}=62,50 \%)$;

3 : espèces recouvrant 25 à $50 \%$ de la superficie du relevé $(\mathrm{RM}=37,5 \%)$;

2 : espèces recouvrant 5 à $25 \%$ de la superficie du relevé $(\mathrm{RM}=15 \%)$;

1 : espèce recouvrant 1 à $5 \%$ de la superficie du relevé $(\mathrm{RM}=2,5 \%)$;

+ : espèces recouvrant moins de $1 \%$ de la superficie du relevé $(\mathrm{RM}=0,2 \%)$;

$r$ : espèces rares $(\mathrm{RM}=0,1 \%)$.

\section{Analyse des données}

Les données ont été analysées à l'aide du Logiciel Excel 2016. Le degré d'hydromorphie du sol, la richesse spécifique, le recouvrement moyen, les indices de diversités de Shannon et de Simpson, l'équitabilité de piélou et les types biologiques ont été considérés comme principaux paramètres de recherche.

Le degré d'hydromorphie du sol a été estimé suivant l'échelle proposée par Senterre (2005) $: 1=$ faible, sol bien drainant, se ressuyant vite après les pluies ; $2=$ moyen, sol ne se ressuyant pas vite après les pluies ; $3=$ fort, sol périodiquement inondé restant longtemps saturé en eau après les pluies ; 4 = permanent, sol saturé en eau même en dehors de la saison des pluies.

La richesse spécifique correspond au nombre d'espèces que compte une communauté ou un peuplement (Ramade, 1994). Pour chaque clairière, le nombre total d'espèces inventoriées a été déterminé.

La fréquence relative ( $\mathrm{Fr}$ ) est le rapport exprimé en pourcentage du nombre de relevés où l'espèce est présente au nombre total des relevés.

$\mathrm{Fr}=100 * \frac{f i}{N}$

Avec $\boldsymbol{f i}:$ nombre de relevés d'inventaire dans lesquels l'espèce i apparaît et $\mathrm{N}$ le nombre total de relevés.

Le recouvrement moyen $(\mathrm{RM})$ est calculé suivant la formule suivante :

$\mathbf{R M}=\frac{\sum \mathbf{R i}}{\mathbf{N}}$

Avec Ri : recouvrement de l'espèce i dans le relevé i et $\mathbf{N}$ : nombre total de relevés.

Indice de diversité de Shannon-Weaver (1949) (H') permet de mesurer les possibilités d'interaction entre les espèces qui composent une communauté. Cet indice varie à la fois en fonction du nombre d'espèces présentes et en fonction de la proportion relative du recouvrement des diverses espèces. Il est compris entre 0,5 bit (indice de diversité très faible) et 4,5 bits environ.

$$
\mathbf{H}^{\prime}=-\sum(\mathbf{n i} / \mathbf{N}) \log _{2}(\mathbf{n i} / \mathbf{N})
$$


ni est le recouvrement moyen d'une espèce $i$

et $\mathrm{N}$ le recouvrement total de toutes les espèces.

L'indice d'équitabilité de Pielou (1961) (E) exprime la régularité, la répartition équitable des espèces au sein d'une communauté. Cet indice qui varie de 0 à 1 est maximal quand les espèces ont des abondances identiques dans le peuplement et il est minimal quand une seule espèce domine tout le peuplement.

\section{$\mathbf{E}=\mathbf{H}^{\prime} / \log _{2} \mathbf{S}$}

$\mathbf{S}$ est le nombre total d'espèces relevées et $\mathbf{H}$ ' l'indice de Shannon.

L'indice de diversité de Simpson (D') : c'est la probabilité pour que deux individus tirés au hasard soient d'espèces différentes. Il est représenté par la réciproque de l'indice de Simpson (D). Le maximum de diversité est représenté par la valeur 1 et le minimum de diversité par la valeur 0 (Danais, 1982).

$\mathbf{D}^{\prime}=\mathbf{1}-\mathbf{D}=\mathbf{1}-\sum(\mathbf{n i} / \mathbf{N})^{2}$

ni est le recouvrement moyen d'une espèce, $\mathrm{N}$ le recouvrement total de toutes les espèces et D l'Indice de Simpson.

Le coefficient de SØrensen (Cs) a permis d'établir les similitudes entre les clairières étudiées sur la base des espèces et leur composition floristique. C'est l'un des indices largement utilisés pour établir les similitudes entre deux sites différents basés sur leur fond floristique (Magurran, 2004). La similarité est basée sur la présence ou l'absence de certaines espèces dans les deux sites. Le coefficient de Sorensen est inférieur à 0,5 si la similitude entre les sites est faible et il est supérieur à 0,5 si les deux sites sont proches. Il est donné par la formule suivante :

$\mathbf{C s}=\frac{2 \mathbf{a}}{2 \mathbf{a}+\mathbf{b}+\mathbf{c}}$

$\mathrm{a}=$ nombre d'espèces communes aux deux clairières ; $b=$ nombre d'espèces de la clairière 1 mais absentes de la clairière 2 et $\mathrm{c}=$ nombre d'espèces de la clairière 2 mais absentes de la clairière 1.

\section{Les types biologiques}

L'examen des types biologiques permet de déterminer les stratégies adaptatives ainsi que la physionomie et la structure de la végétation. Les principaux types biologiques utilisés dans cette étude sont basés sur la classification de Raunkiaer (1934) modifié par Lebrun (1947). On distingue donc les Thérophytes, les Hémicryptophytes, les Géophytes, les Hydrophytes, les Chaméphytes et les Phanérophytes. Le spectre biologique brut tient compte simplement du nombre d'espèces relevant d'un type biologique donné tandis que le spectre biologique pondéré est construit à partir de la pondération des espèces par le recouvrement moyen.

Tableau 1: Paramètres dimensionnels des clairières.

\begin{tabular}{lccccc}
\hline Clairières & Superficie $\left(\mathbf{m}^{2}\right)$ & $\begin{array}{c}\text { Surface } \\
\text { échantillonnée }\left(\mathbf{m}^{2}\right)\end{array}$ & $\begin{array}{c}\text { Nombre de } \\
\text { transects }\end{array}$ & $\begin{array}{c}\text { Nombre de } \\
\text { parcelles }\end{array}$ & $\begin{array}{c}\text { Nombre de } \\
\text { relevés }\end{array}$ \\
\hline Ndangayé & 200000 & 510 & 10 & 102 & 510 \\
Djangui & 100080 & 255 & 25 & 50 & 250 \\
Djaloumbé & 18200 & 50 & 5 & 10 & 50 \\
Petite savane & 17692 & 40 & 4 & 8 & 40 \\
\hline
\end{tabular}



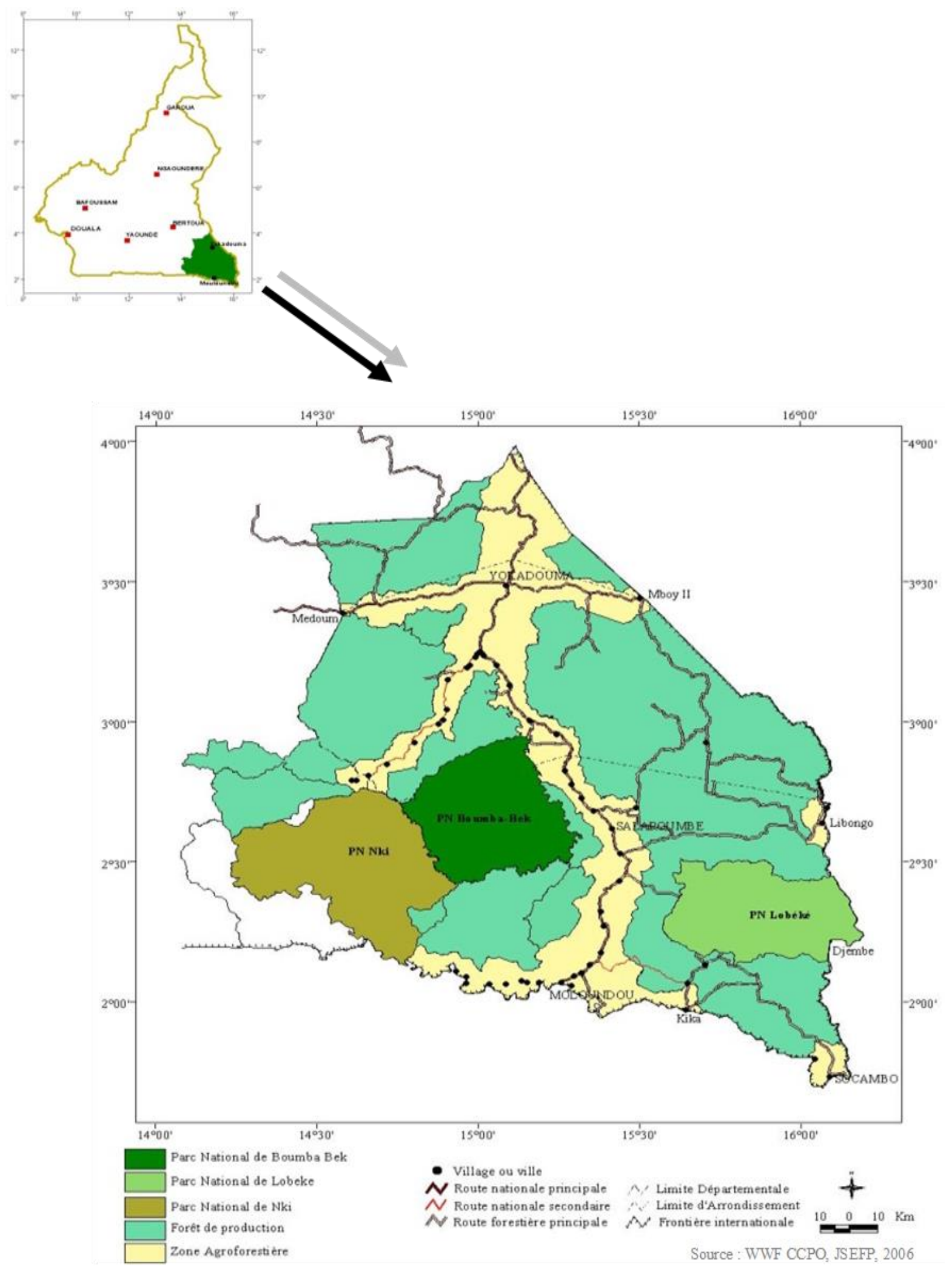

Figure 1: Localisation du parc National de Lobéké dans le domaine forestier du Sud-Est Cameroun. 

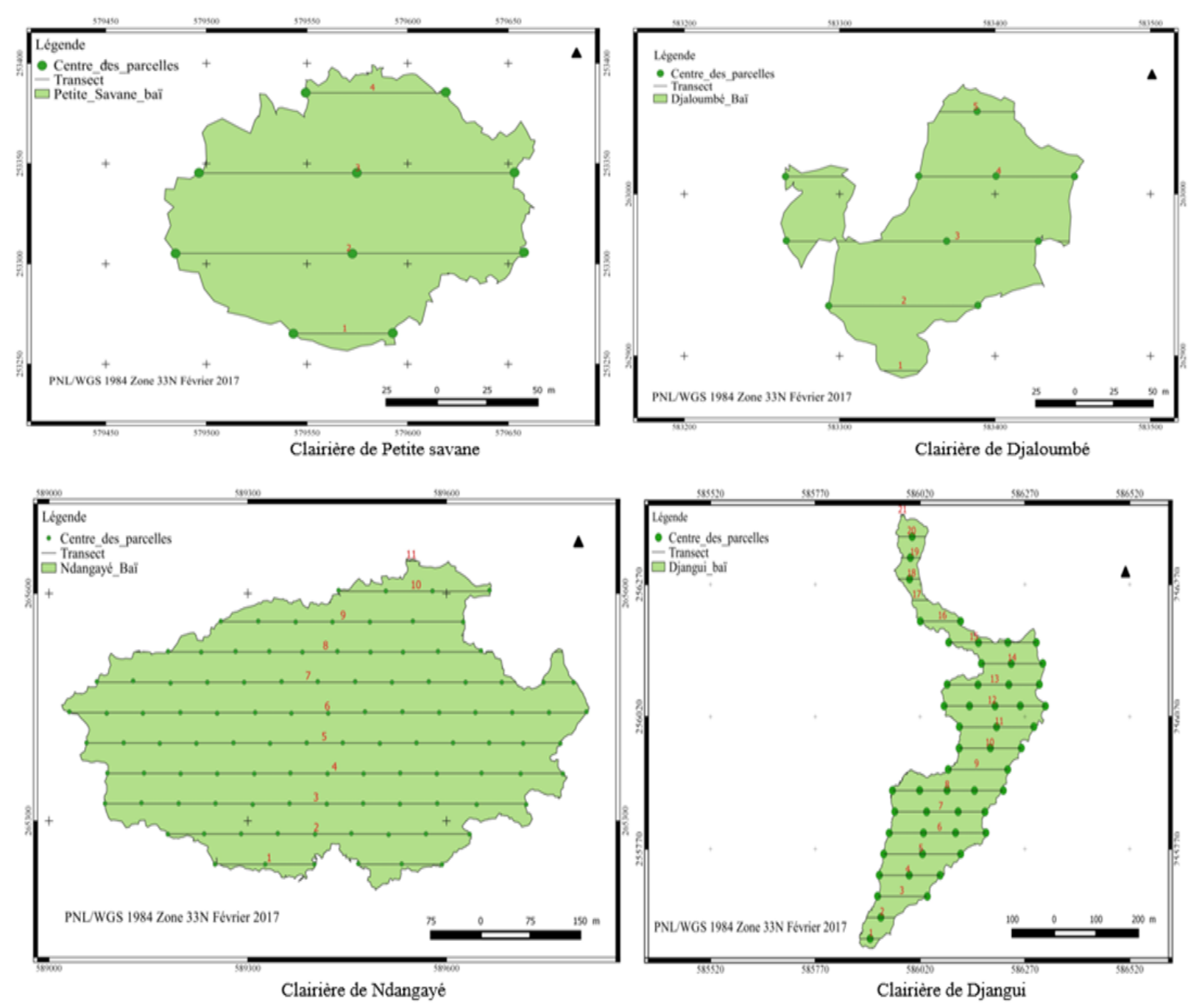

Figure 2 : Plans d'échantillonnage des clairières.

\section{RESULTATS}

\section{Richesse et diversité floristique des clairières}

Dans l'ensemble, le cortège végétal des clairières étudiées fait état de 70 espèces végétales réparties entre 60 genres appartenant à 29 familles. Le Tableau 2 présente les caractéristiques de chaque clairière.

Il ressort du Tableau 2 que la clairière de Djangui possède la plus grande richesse spécifique avec 46 espèces végétales tandis que la clairière de Djaloumbé présente la plus faible richesse spécifique avec 30 espèces. Djangui est également la clairière la plus diversifiée car présente les indices de diversités les plus élevés. Le nombre de familles botaniques dans les clairières est sensiblement le même, entre 17 et 18 . Soixante- deux (62) des espèces recensées sont des herbacées et 8 des espèces ligneuses. La Figure 3 présente le nombre d'espèces par famille.

Les familles les plus représentées en espèces sont les Cyperaceae (13 espèces), Poaceae ( 9 espèces), Fabaceae (6 espèces), Asteraceae (5 espèces), Acanthaceae (4 espèces) et Rubiaceae (3 espèces). Un autre groupe est constitué de 7 familles représentées 
par deux espèces chacune (Commelinaceae, Lamiaceae, Marantaceae, Malastomataceae, Onagraceae, Malvaceae et Rubiaceae et Moraceae). Plus de la moitié des familles (dix-sept) est caractérisée par la présence d'une seule espèce. Ce sont les familles des Anacardiaceae, Apiaceae, Apocynaceae, Arecaceae, Asteraceae, Caryophyllaceae, Combretaceae, Cucurbitaceae, Ebenaceae, Euphorbiaceae, Nympheaceae, Oxalidaceae, Euphorbiaceae,

Plantaginaceae, Thelypteridaceae et Zingiberaceae.

Les fréquences relatives d'apparition des espèces dans chaque clairière sont présentées par les Figures 3, 4, 5 et 6 . A Ndangaye (Figure 4), Rhynchospora corymbosa est l'espèce la plus observée avec une fréquence de $94,3 \%$, suivie d'Echinochloa crus-pavonis $(90,8 \%)$, d'Oxalis sp. $(81,4 \%)$ et de Pentodon pentandrus (67,1\%). A Djangui (Figure 5) Rhynchospora corymbosa occupe également la première place avec une fréquence de $35,6 \%$. Elle est suivie de Ludwigia abyssinica $(27,6 \%)$ et de Pentodon pentandrus $(22,8 \%)$. A petite savane (Figure 6), les espèces les plus fréquentes sont Paspalum conjugatum (20\%), Commelina sp. $(17,5 \%)$ et Pentodon Pentandrus (15\%). La clairière de Djaloumbé (Figure 7), quant à elle, est caractérisée par la quasi-présence dans tous les relevés de Mimosa pudica (96,4\%), suivie d'Acroceras amplectens avec une fréquence de 52\%, de Commelina sp. (38\%) et de Paspalum conjugatum (38\%).

Seulement 9 espèces $(13,05 \%)$ se sont retrouvées dans toutes les quatre clairières. Il s'agit de Rhynchospora corymbosa, Cyperus difformis, Acroceras amplectens, Paspalum conjugatum, Pentodon pentandrus, Acmela caulirhiza, Commelina sp., Desmodium adscendens et Hydrocharis chevalieri.

\section{Taux de recouvrement des clairières}

Les taux de recouvrement présentés par le Tableau 3 permettent de mieux apprécier la dominance des espèces dans les clairières. Les familles dominantes sont les Cyperaceae, les Poaceae et les Rubiaceae. La végétation de Ndangaye est dominée par Rhynchospora corymbosa qui a un taux de recouvrement de
$23,3 \%$, suivie par Oxalis sp. $(19,60 \%)$ et Echinochloa crus-pavonis (13,78\%). La clairière de Djangui est dominée par Acroceras amplectens (12,80\%), Paspalum conjugatum $(12,02 \%)$ et Rhynchospora corymbosa (10,7\%). A Petite Savane, Pentodron pentandus et Paspalum conjugatum se partagent le haut du podium, suivies de Rhynchospora corymbosa et Enydra fluctuans. A Djaloumbé, la Fabaceae Mimosa pudica (Figure 8) occupe à elle seule $73 \%$ de la clairière.

\section{Similitude entre les clairières}

Le Tableau 4 donne les valeurs du coefficient de similitude de SØrensen entre les différentes clairières.

Les comparaisons entre les clairières montrent que le couple de clairières DjanguiNdangaye présente le plus fort coefficient de similitude $(0,70)$, suivi des couples Petite Savane-Ndangaye $(0,58)$ et Djangui-Petite Savane $(0,56)$. Ces valeurs supérieures à 0,50 signifient que les fonds floristiques de ces trois clairières sont proches. Par ailleurs, les plus faibles valeurs s'observent lorsqu'on compare les sites de Djaloumbé avec les trois autres clairières. Ce qui indique que le fond floristique de Djaloumbé est différent de celui des autres sites

\section{Types biologiques}

Le spectre biologique (Figure 8) représente la portion de chaque type biologique constituant le couvert végétal.

Les proportions des types biologiques varient d'une clairière à l'autre. Pour les clairières Djangui, Ndangaye et Petite Savane, les géophytes (22 à $34 \%$ ) et les thérophytes (22 à 39\%) sont les formes de vies les plus abondantes (spectres bruts). Ils sont également les formes de vie les plus dominantes avec un recouvrement moyen de 26 à $59 \%$ pour les géophytes et de 35 à $38 \%$ pour les thérophytes. On note une très faible fréquence des hydrophytes et des hémicryptophytes. La clairière Djaloumbé présente cependant des spectres très différents de ceux des autres clairières avec une forte dominance des phanérophytes (78\%). 
Tableau 2 : Richesse spécifiques et Indices de diversités des différentes clairières du PNL.

\begin{tabular}{llllllll}
\hline & $\begin{array}{l}\text { Degré } \\
\text { humidité }\end{array}$ & $\begin{array}{l}\text { Nombre } \\
\text { familles }\end{array}$ & $\begin{array}{l}\text { Nombre } \\
\text { genres }\end{array}$ & $\begin{array}{l}\text { Nombre } \\
\text { espèces }\end{array}$ & Shannon & Piélou & Simpson \\
\hline Djaloumbé & Moyen & 17 & 29 & 30 & 1,61 & 0,33 & 0,55 \\
Djangui & Fort & 18 & 39 & 46 & 3,99 & 0,73 & 0,92 \\
Ndangayé & Fort & 18 & 34 & 40 & 3,27 & 0,62 & 0,86 \\
$\begin{array}{l}\text { Petite } \\
\text { Savane }\end{array}$ & Fort & 18 & 29 & 32 & 3,49 & 0,67 & 0,90 \\
\hline
\end{tabular}

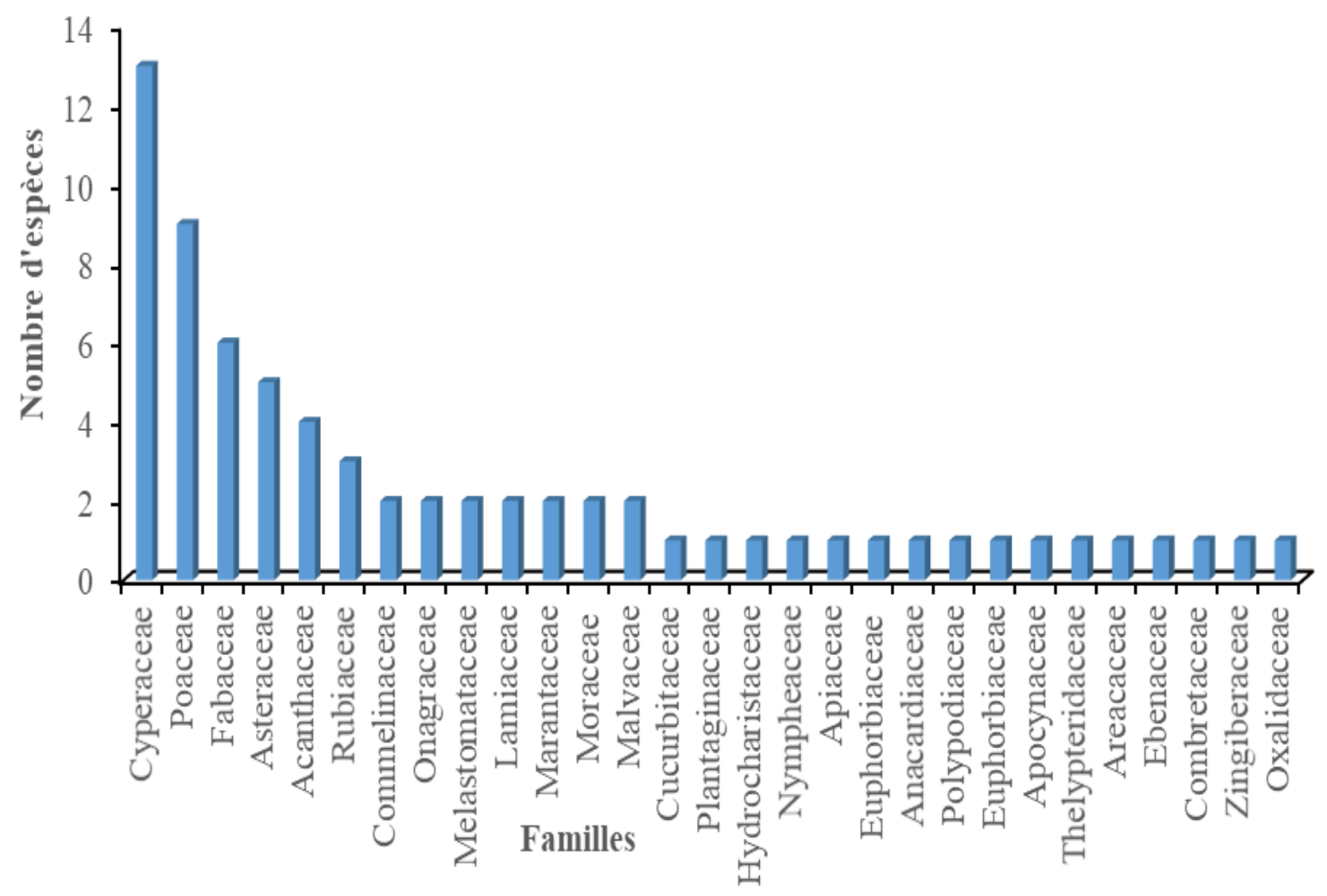

Figure 3 : Répartition des espèces végétales par familles. 


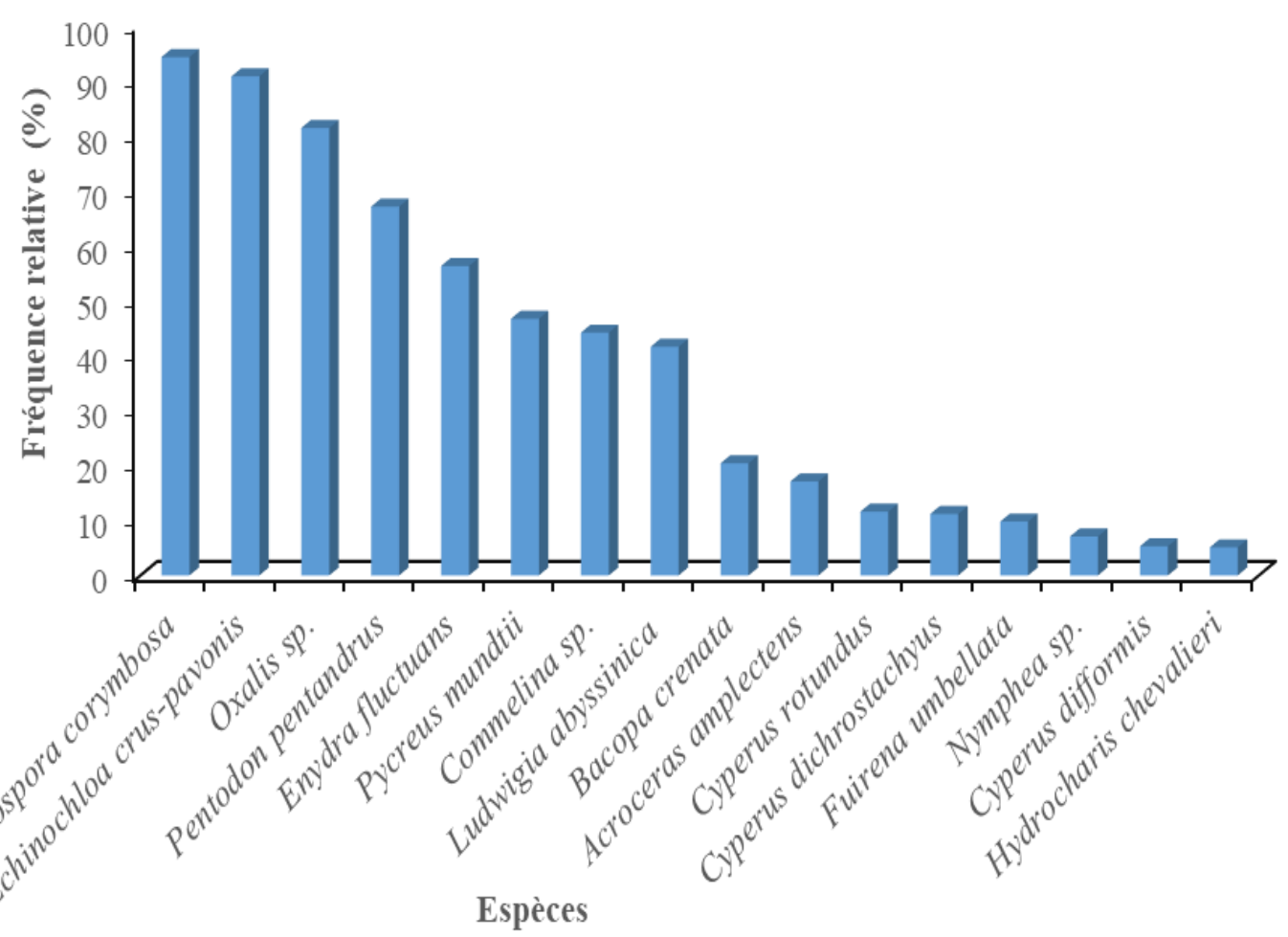

Figure 4 : Espèces ayant plus de 5\% de fréquence relative dans la clairière de Ndangayé.
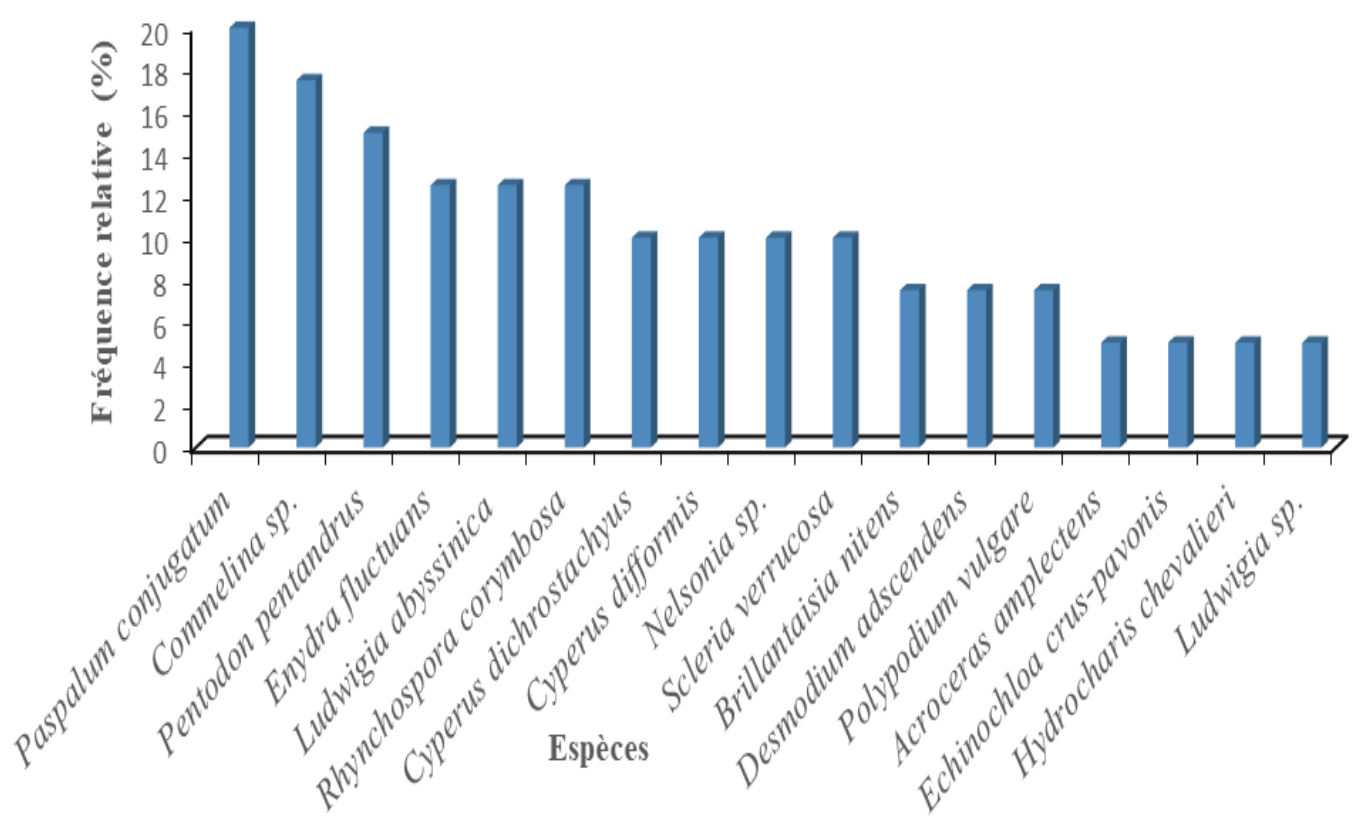

Figure 5 : Espèces ayant plus de 5\% de fréquence relative dans la clairière de Djangui. 


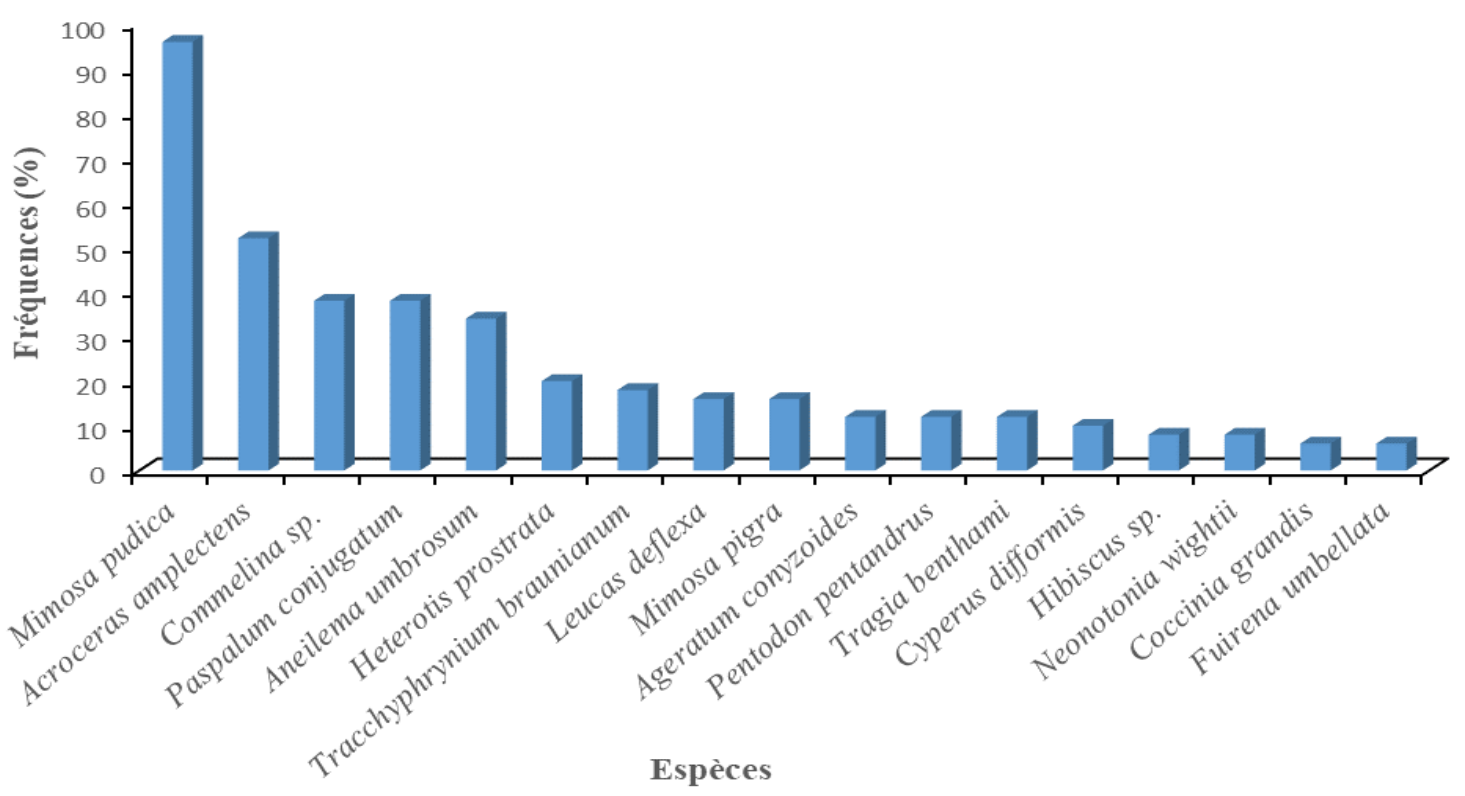

Figure 6: Espèces ayant plus de 5\% de fréquence relative dans la clairière de Petite Savane.
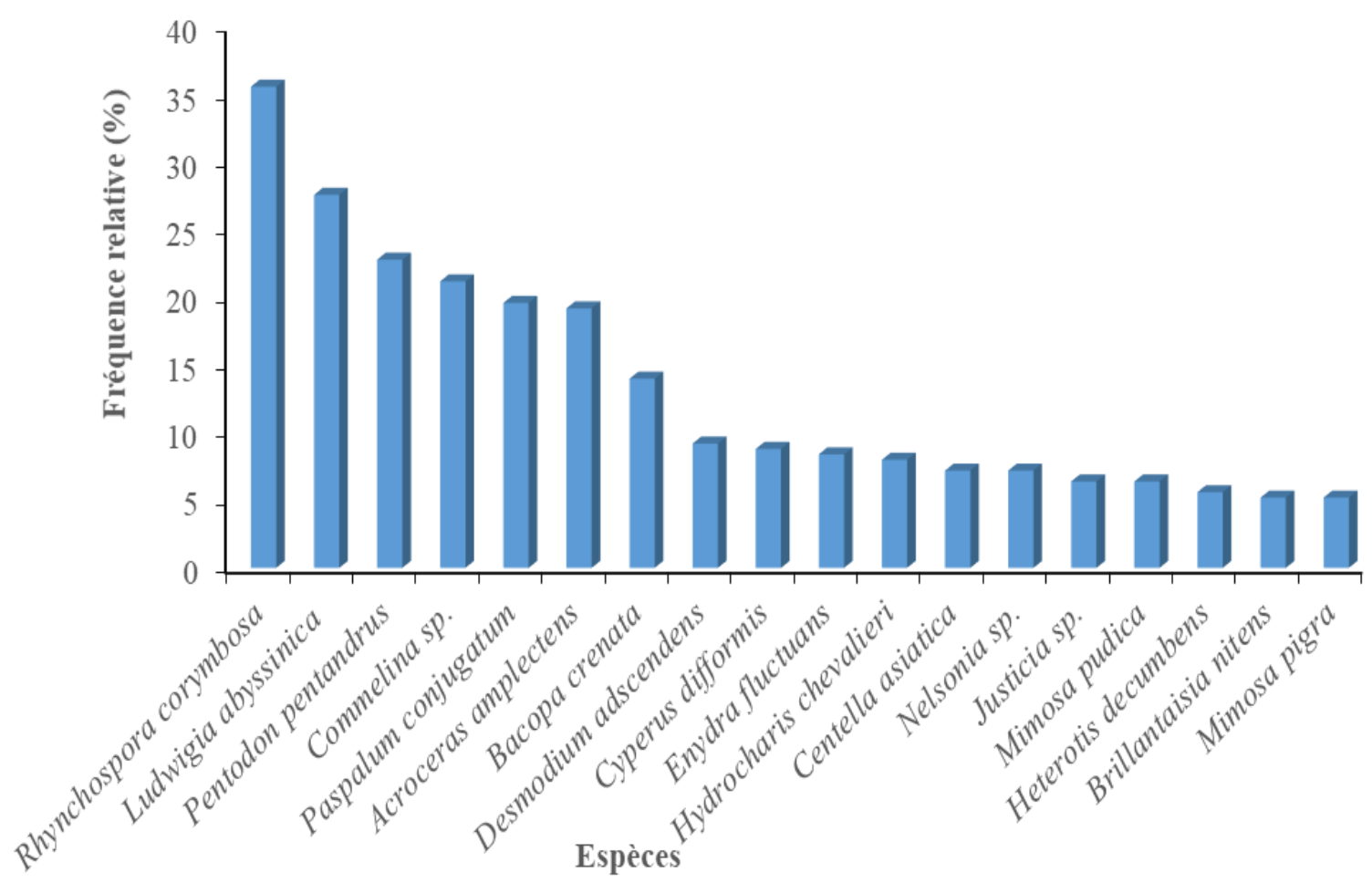

Figure 7: Espèces ayant plus de 5\% de fréquence relative dans la clairière de Djaloumbé. 
Tableau 3: Taux de recouvrement des espèces dominantes des clairières.

\begin{tabular}{|c|c|c|c|}
\hline Clairières & Espèces dominantes & Familles & Recouvrement Moyen (\%) \\
\hline & Rhynchospora corymbosa & Cyperaceae & 23,36 \\
\hline & Oxalis sp. & Oxylidaceae & 19,60 \\
\hline \multirow[t]{7}{*}{ Ndangayé } & Echinochloa crus-pavonis & Poaceae & 13,78 \\
\hline & Enydra fluctuans & Asteraceae & 13,33 \\
\hline & Pycreus mundtii & Cyperaceae & 7,20 \\
\hline & Pentodon pentandrus & Rubiaceae & 6,90 \\
\hline & Autres & & 15,83 \\
\hline & Acroceras amplectens & Poacaea & 12,80 \\
\hline & Paspalum conjugatum & Poaceae & 12,03 \\
\hline \multirow[t]{8}{*}{ Djangui } & Ludwigia abyssinica & Onagraceae & 10,80 \\
\hline & Rhynchospora corymbosa & Cyperaceae & 10,70 \\
\hline & Pentodon pentandrus & Rubiaceae & 9,60 \\
\hline & Bacopa crenata & Plantaginaceae & 8,65 \\
\hline & Commelina sp. & Commelinaceae & 5,50 \\
\hline & Autres & & 25,37 \\
\hline & Pentodon pentandrus & Rubiaceae & 16,90 \\
\hline & Paspalum conjugatum & Poaceae & 16,50 \\
\hline \multirow[t]{7}{*}{ Petite Savane } & Enydra fluctuans & Asteraceae & 10,50 \\
\hline & Ludwigia abyssinica & Onagraceae & 9,02 \\
\hline & Scleria verrucosa & Cyperaceae & 8,50 \\
\hline & Rhynchospora corymbosa & Cyperaceae & 8,40 \\
\hline & Cyperus difformis & Cyperaceae & 6,10 \\
\hline & Autres & & 24,08 \\
\hline & Mimosa pudica & Fabaceae & 73,40 \\
\hline \multirow[t]{4}{*}{ Djaloumbé } & Commelina sp. & Commelinaceae & 6,46 \\
\hline & Pentodon pentandrus & Rubiaceae & 5,03 \\
\hline & $\begin{array}{l}\text { Tracchyphrynium } \\
\text { braunianum }\end{array}$ & Marantaceae & 5,36 \\
\hline & Autres & & 9,72 \\
\hline
\end{tabular}

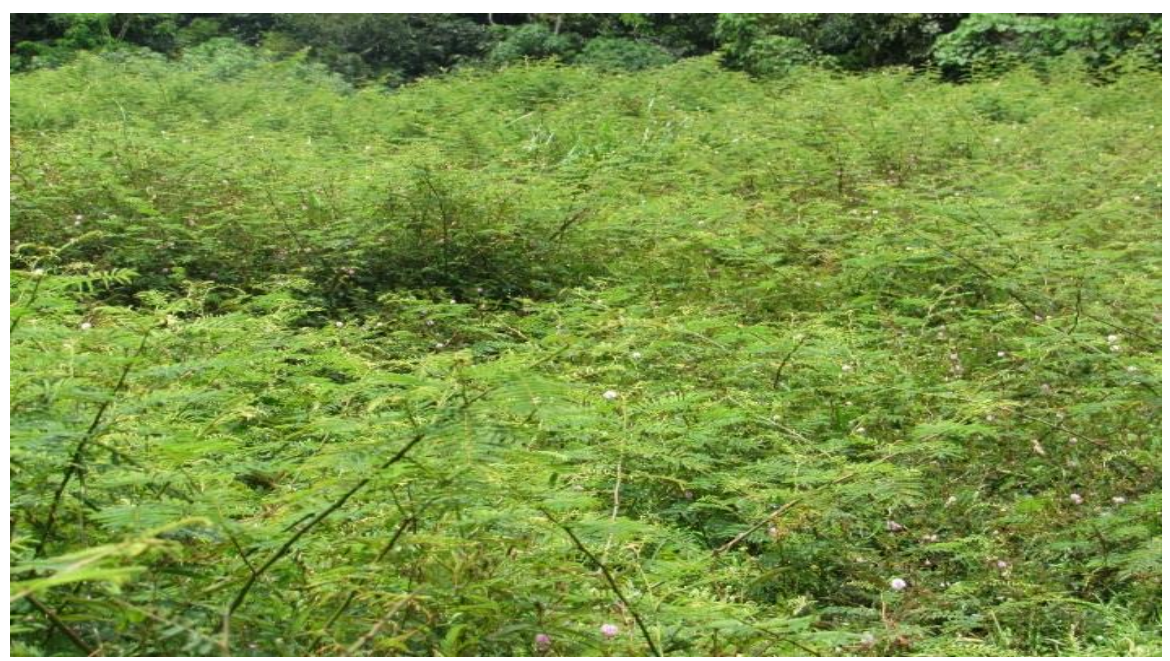

Figure 8 : Fourré de Mimosa pudica dans la clairière Djaloumbé. 


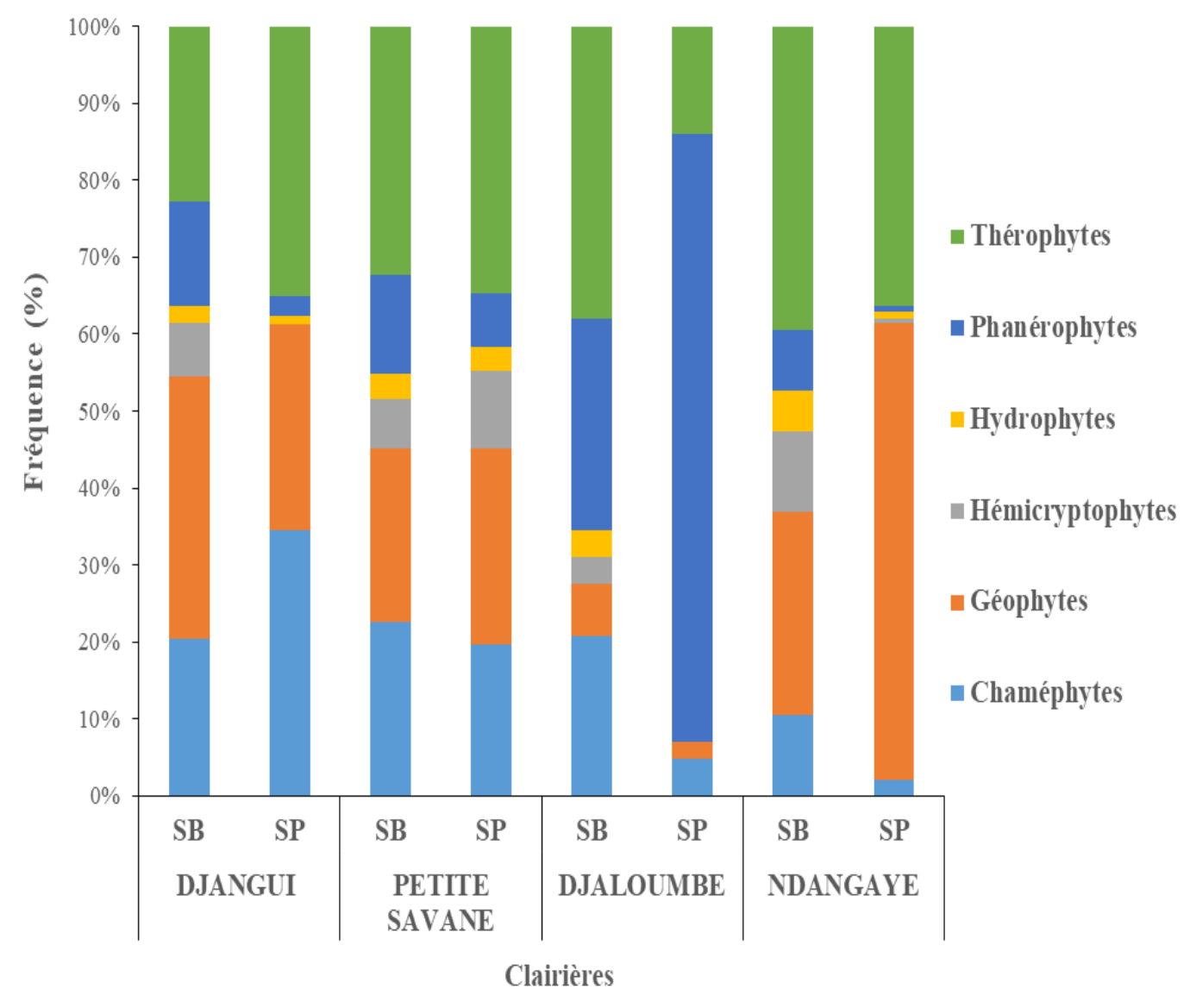

Légende: $\mathrm{SB}=$ Spectre Brut. SP = Spectre Pondéré

Figure 8: Spectres brut et pondéré des types biologiques des clairières du Parc National de Lobéké.

Tableau 4: Valeurs de l'indice de similitude de SØrensen.

\begin{tabular}{lcccc}
\hline & Djangui & Ndangayé & Petite savane & Djaloumbé \\
\hline Djangui & 1 & 1 & & \\
Ndangayé & 0,70 & 0,58 & 1 & \\
Petite savane & 0,56 & 0,46 & 0,32 & 1 \\
Djaloumbe & 0,50 & & & \\
\hline
\end{tabular}




\section{DISCUSSION}

Les clairières représentent, à l'intérieur des forêts tropicales des habitats distincts dont la végétation diffère considérablement de celle des habitats voisins. Dans les 4 clairières étudiées, 70 espèces appartenant à 29 familles ont été recensées. Le nombre d'espèces par clairière varie de 30 à 46 , ce qui est proche des 45 espèces recensées par Magliocca (2000) dans la clairière de Maya Nord au Congo, mais inférieur au nombre d'espèces (91) identifiées par Boupoya et al. (2010) dans la clairière marécageuse de Mekandjé dans le Parc National de l'Ivindo au Gabon. Il convient cependant de signaler que ces derniers auteurs ont réalisé des relevés dans la lisière, ce qui n'a pas été le cas dans cette étude.

Les indices de diversité de Shannon et de Simpson indiquent non seulement la richesse spécifique, mais prennent aussi en compte la régularité des espèces. Ainsi considérant le nombre d'espèces et les indices, la diversité est plus grande à Djangui et plus faible à Djaloumbé. Cette situation à Djaloumbé s'explique par le fait que la clairière est recouverte à $73 \%$ par une seule espèce Mimosa Pudica, les 29 autres espèces se partageant $27 \%$ de recouvrement. Les indices de diversité de Shannon compris entre 1,61 et 3,99 sont inférieurs à la moyenne de 4,38 trouvée par Boupoya (2010) dans le Parc National de l'Ivindo.

Les clairières marécageuses du PNL sont des zones à végétation presque exclusivement herbacée $(88,57 \%)$. Les familles dominantes sont les Cyperaceae et les Poaceae avec 22 espèces au total, soit $31,92 \%$ des espèces dans le cortège floristique global. Ceci corrobore les résultats de Magliocca (2000) dans le parc national d'Odzala et ceux de Boupoya (2010) dans le Parc National de l'Ivindo qui ont trouvé les mêmes familles dominantes.

Bien que la grande majorité des familles trouvées à Lobéké soient globalement les mêmes que celles trouvées par Boupoya et al. (2010) dans la clairière de Mékandjé dans le Parc National de l'Ivindo au Gabon, il existe une grande différence dans les genres et les espèces. En effet, seules dix espèces sont communes à cette clairière et celles du Parc National de Lobéké parmi lesquelles Rhynchospora corymbosa, Pycreus mundtii, Bocapa crenata, Fuerena umbellata, Ludwigia abyssinica... La composition floristique des clairières hydromorphes du PNL est également très différente de celle des clairières sur schiste des parcs nationaux de Boumba-Bek et de Nki au Sud-Est Cameroun décrite par Noupa et Nkongmeneck (2008b). Les deux types de clairières partagent en commun seulement trois espèces, ceci est probablement dû aux conditions pédologiques complètement différentes dans ces sites.

Dans le PNL, la flore est assez homogène d'une clairière à une autre, avec de petites particularités pour certaines clairières. Les indices de similitude de SØrensen révèlent que Djangui, Petite Savane et Ndangaye ont un même fond floristique. Ces trois sites pourraient être soumis aux mêmes conditions du milieu et donc leur végétation varie peu. Djaloumbé, par contre, a présenté des coefficients de similitude faibles avec les autres clairières. Ces clairières sont géographiquement proches donc cette différence ne peut s'expliquer que par les conditions pédologiques et d'engorgement du sol qui influencent la végétation de chaque clairière. En effet, la clairière de Djaloumbé possède un sol argilo-sablonneux avec un degré d'humidité moyen, contrairement aux trois autres sites dont les sols, avec un degré d'humidité fort sont périodiquement inondés, restant longtemps saturés en eau après les pluies.

La famille des Cyperaceae est la plus abondante avec un pourcentage de $18,84 \%$ des espèces. Bien que certaines parties des espèces de cette famille (Rhynchospora corymbosa, Pycreus mundtii, etc...) soient consommées par la faune (Metsio Sienne et al., 2014), les Cyperaceae constituent la végétation herbacée haute, avec des feuilles tranchantes qui peuvent causer des blessures aux animaux et ainsi conduire à une baisse de la fréquentation des clairières.

La présence des fourrés épineux de Mimosa pudica à Djaloumbé pourrait traduire 
une fermeture de cette clairière. A côté de cette espèce qui occupe $73 \%$ de la surface de la clairière, on retrouve également la Marantaceae Tracchyphrynium braunianum (5\% de recouvrement) qui, dans la plupart du temps, n'est présente qu'en lisière des clairières. De même, le spectre biologique montre que la clairière de Djaloumbé est atypique avec une dominance de phanérophytes. Dans les autres clairières du parc, les géophytes et les thérophytes sont les formes de vies dominantes, ce qui corrobore les résultats de Boupoya et al. (2010) à Mekandjé dans le Parc National de l'Ivindo au Gabon. La prépondérance des géophytes et des thérophytes dans les clairières est liée aux conditions d'hydromorphie qui règnent dans ce milieu. La vase ainsi que les piétinements des animaux ne permettent pas l'installation d'espèces phanérophytiques. Cet impact des mammifères sur la végétation des clairières a été mentionné par plusieurs auteurs dont Magliocca et Gautier-Hion (2001) et Vande Weghe (2006). La dominance des espèces phanérophytiques dans la clairière de Djaloumbé et donc son embroussaillement serait due à la faible fréquentation de celle-ci par la grande et moyenne faune.

\section{Conclusion}

Les clairières sur sol hydromorphe du Parc National de Lobéké présentent un cortège de 70 espèces végétales. Dans l'ensemble, ces clairières ont une faible diversité et sont dominées par les espèces de la famille des Cyperaceae, des Poaceae et des Fabaceae. Les géophytes et les thérophytes sont les formes de vies dominantes à l'exception de la clairière de Djaloumbé dominée par les espèces phanérophytiques. Il est nécessaire de conduire des études dans l'ensemble des clairières du parc pour mieux comprendre la relation entre leur fréquentation par la grande et la moyenne faune et la dynamique de la végétation. Des actions d'enlèvement de Mimosa pudica et des espèces de Cyperaceae envahissantes doivent être envisagées dans le cadre de l'aménagement de ces clairières.

\section{CONFLIT D'INTERETS}

Les auteurs ne déclarent aucun conflit d'intérêts.

\section{CONTRIBUTIONS DES AUTEURS}

TLF a contribué à l'élaboration du protocole de recherche, à la collecte et à l'analyse des données et à la rédaction du manuscrit. MSMC a contribué à l'analyse des données et à la rédaction du manuscrit. MEMV a contribué à la collecte des données. MNA a encadré le travail sur le terrain.

\section{REMERCIEMENTS}

Les auteurs remercient le service de conservation du Parc National de Lobéké pour l'appui logistique octroyé pour la réalisation de cette étude.

\section{REFERENCES}

Braun-Blanquet J. 1932. Plant Sociology: The Study of Plant Communities. Mc Graw Hill: New York and London.

Boupoya A, Doumenge C, Lejoly J. 2010. La végétation des clairières sur sol hydromorphe dans le massif forestier du Nord-Est du Gabon : Premières études sur la clairière de Mékandjé (Parc National de l'Ivindo). Acta Botanica Gallica. 157(1): 135-150. https://doi.org/10.1080/12538078.2010.1 0516195

Boupoya A. 2010. Flore et végétation des clairières intra-forestières sur sol hydromorphe dans le Parc National de l'Ivindo (Nord-Est Gabon). Thèse de Doctorat, Université Libre de Bruxelles. $283 \mathrm{p}$.

Danais M. 1982. La diversité en écologie : Analyse bibliographique. Botanica Rhedonica, A., 17: 77-104.

Dufrêne M. 2003. Méthodes d'analyse des données écologiques et biogéographiques. Cours Agro 044Bioingénieur, Université Libre de Bruxelles.

Ekobo A. 1995. Conservation of the African forest elephant (Loxodonta africana cyclotis) in Lobéké, Southeast 
Cameroon. PhD. thesis, University of Kent. 151 p.

Eno Nkuh M. 2011. Frequency of large mammals visits to forest clearing for ecotourism potentials in the CampoMa'an National Park, Southern Cameroon. VDM Verlag.

Gatti S, Levréro F, Menard N, Gauthier-Hion A. 2004. Population and group structure of western lowland gorillas at Lokoué Republic of Congo. Am. J. Primatol., 68: 111-123. DOI: $10.1002 / a j p / 20045$.

Gessner J, Buchwald R, Wittemyer G. 2013. Assessing species occurrence and species-specific use patterns of bais (forest clearings) in Central Africa with camera traps. Afr. J. Ecol., 52(1): 59-68. https://doi.org/10.1111/aje.12084

Harris D. 1999. Lobéké: Botanical Inventory. WWF Cameroon Technical Report. 130p.

Laclavère G. 1979. Atlas de la République Unie $d u$ Cameroun. Jeune Afrique: Paris, 72p.

Lebrun J. 1947. La végétation de la plaine alluviale au sud du Lac Edouard. Institut des Parcs Nationaux du Congo Belge, exploration du Parc National Albert. Mission Lebrun (1937-1938). Fascicule, 1: $472-800$.

Letouzey R. 1985. Notice explicative de la carte phytogéographique du Cameroun au 1/500.000. Encyclopédie Biologique 69, Edition Paul Le Chevalier : Paris.

Magliocca F. 2000. Etude d'un peuplement de grands mammifères forestiers tropicaux fréquentant une clairière : structure des populations ; utilisation des ressources ; coexistence intra- et interpopulationnelle. Thèse de Doctorat, Université de Rennes 1. 285 p.

Magliocca F, Gautier-Hion A. 2001. Les clairières en forêts tropicales : des aires à protéger en toute priorité. Canopée, 20: $1-9$.

Magliocca F, Gautier-Hion A. 2002. Mineral content as a basis for food selection by western lowland gorillas in a forest clearing. Am. J. Primatol., 57: 67-77. https://doi.org/10.1002/ajp.10034
Magurran AE. 2004. Measuring Biological Diversity. Blackwell Science Ltd: London.

Metsio Sienne J, Buchwald R, Wittemyer G. 2013. Differentiation in mineral constituents in Elephant selected versus unselected water and soil resources at Central African bais (forest clearings). Eur. J. Wildl. Res., 60(2): 377-382. https://doi.org/10.1007/s10344-0130781-0

Metsio Sienne J, Buchwald R, Wittemyer G. 2014. Plant mineral concentrations related to foraging preferences of western lowland gorilla in Central African forest clearings. Am. J. Primatol., $\quad$ 76: 1115-1126. https://doi.org/10.1002/ajp.22297

Mindonga-Nguelet FD, Zinga CR, Midoko D, Nzengue E, Mikala C, Okouyi J, Mbachi B, Mavoungou JF, Shongo M. 2016. Identification et caractérisation de la dynamique de la grande faune dans le baï de Momba (nord-est Gabon). Int. J. Biol. Chem. Sci., 10(3): 1037-1047. http://dx.doi.org/10.4314/ijbcs.v10i3.11

Noupa P, Nkongmeneck BA. 2008a. Influence des clairières forestières sur la répartition spatiale des grands mammifères dans la forêt dense du bassin du Congo: Cas du Parc National de Boumba-Bek (Sud-Est Cameroun). Int. J. Biol. Chem. Sci., 2(2): 185-195.

https://dx.doi.org/10.4314/ijbcs.v2i2.397 34

Noupa P, Nkongmeneck BA. 2008b. Evaluation de la diversité floristique de six clairières sur schiste dans le massif forestier de Boumba-Bek et Nki (Sud-Est Cameroun). Int. J. Biol. Chem. Sci., 2(4): 529-548.

https://dx.doi.org/10.4314/ijbcs.v2i4.397 64

Nzooh Z. 2003. Suivi écologique dans le Parc National de Lobéké et sa zone périphérique : données de base sur la dynamique des populations de grands et moyens mammifères et des activités anthropiques. Rapport Technique WWF CCPO. 205p. 
Piélou EC. 1966. Species diversity and pattern diversity in the study of ecological succession. J. Theor. Biol., 10: 370-383.

Ramade F. 1994. Éléments d'Ecologie. Écologie Fondamentale 2. Ediscience International: Paris.

Raunkiaer C. 1934. The Life Forms of Plants and Statistical Plant Geography. Oxford University Press: Oxford.

Senterre B. 2005. Recherches méthodologiques pour la typologie de la végétation et la phytogéographie des forêts denses d'Afrique tropicales. Thèse de Doctorat. Université Libre de Bruxelles. 349 p.

Shannon CE, Weaver W. 1949. The Mathematical Theory of Communication. University of Illinois Press: Urbana.

Sørensen T. 1948. A Method of establishing groups of equal amplitude in plan sociology based on similarity of species content and its application to analysis of vegetation on Danish Common. Kong. Danske. Videns. Selskob. Biolg. Sckr. Kjobenhavn., 4: 1-34.

Temgoua LF. 2006. Identification et caractérisation des clairières marécageuses du parc national de l'Ivindo (Gabon). Mémoire de Master, spécialisation Foresterie Rurale et Tropicale (FRT) ENGREF Montpellier. $80 \mathrm{p}$.
TNS. 2012. Proposition d'inscription sur la liste du patrimoine mondial. Trinational de la Sangha. http://whc.unesco.org/uploads/nominatio ns/1380rev.pdf.

Tsakem SC, Tchamba M, Weladji RB. 2015. Les gorilles du Parc National de Lobéké (Cameroun): Interactions avec les populations locales et implications pour la conservation. Int. J. Biol. Chem. Sci., 9(1): 270-280. http://dx.doi.org/10.4314/ijbcs.v9i1.24

Turkalo AK, Wrege PH, Wittemyer G. 2013, Long-term monitoring of Dzanga baï forest Elephants: Forest clearing use patterns. PLOS ONE 8(12): 1-12. DOI: 0.1371 journal.pone.0085154.

Vande Weghe JP. 2006. Ivindo et Mwagna: Eaux Noires, Forêt Vierge et Baïs. Wildlife Conservation Society: Libreville, Gabon.

White L, Edwards A. 2001. Conservation en Forêt Pluviale Africaine, Méthodes de Recherche. WCS (Wildlife Conservation Society): Libreville, Gabon.

Zinga CR, Midoko D, Nzengue E, Maroundou AP, Zabiti G, Mikala C. 2016. Dynamique de fréquentation des grands mammifères dans une clairière marécageuse: Cas du baï de Momba au Nord-Est du Gabon. J. Appl. Biosci., 103: 9877-9885. http://dx.doi.org/10.4314/jab.v103i1.9 